\title{
Acknowledgement to Referees
}

(c) Springer Nature Switzerland AG 2021

\section{Dear Reader,}

Welcome to the final issue of Molecular Diagnosis \& Therapy for 2021.

I wish to reflect on this year's achievements, and to thank all those who have contributed their time and effort to guarantee the quality of the content published in the journal.

To date, in 2021, over 50 articles have been published. The most popular of these in terms of downloads from SpringerLink have been:

- Current and Emerging Clinical Treatment in Mitochondrial Disease. Tinker, R.J., Lim, A.Z., Stefanetti, R.J. et al. Mol Diagn Ther 25, 181-206

- Evaluating Infectious, Neoplastic, Immunological, and Degenerative Diseases of the Central Nervous System with Cerebrospinal Fluid-Based Next-Generation Sequencing. Tsamis, K.I., Sakkas, H., Giannakis, A. et al. Mol Diagn Ther 25, 207-229

- Luminal Breast Cancer: Risk of Recurrence and Tumor-Associated Immune Suppression. Pellegrino, B., Hlavata, Z., Migali, C. et al. Mol Diagn Ther 25, 409-424

- System-Wide Pollution of Biomedical Data: Consequence of the Search for Hub Genes of Hepatocellular Carcinoma Without Spatiotemporal Consideration. Sharma, A., Colonna, G. Mol Diagn Ther 25, 9-27

- Leveraging the Fragment Length of Circulating Tumour DNA to Improve Molecular Profiling of Solid Tumour Malignancies with Next-Generation Sequencing: A Pathway to Advanced Non-invasive Diagnostics in Precision Oncology?. Underhill, H.R. Mol Diagn Ther 25, 389-408

- Treatable Mechanisms in Asthma. Cazzola, M., Ora, J., Cavalli, F. et al. Mol Diagn Ther 25, 111-121

The high quality of content published in Molecular Diagnosis \& Therapy has been reflected in the most recent impact factor of 4.074 and CiteScore ${ }^{\mathrm{TM}}$ of 6.3. Further, Molecular Diagnosis \& Therapy has published content in a timely manner, with an average time from submission to first decision of 14 days.

The COVID-19 pandemic has continued to present many challenges and I would like to thank all who have contributed to ensuring the journal has thrived despite the ongoing issues.

I would like to start by thanking the authors of the articles published in Molecular Diagnosis \& Therapy over the course of 2021. The enthusiasm of all authors for their chosen fields and their willingness to contribute content to the journal are crucial for its continued success.

The quality of published articles is also testament to the diligence of the peer reviewers. I would like to acknowledge the following individuals who acted as reviewers for Molecular Diagnosis \& Therapy in the last 12 months:

Adam Abate, USA

Mustapha Abubakar, USA

Animesh Acharjee, UK

Chiara Agnoletto, Italy

Sudhir Agrawal, USA

Alexandre Alanio, France

Guillermo Aquino-Jarquin, Mexico
Mehdi Arbabi Ghahroudi, Canada

Sylvia L. Asa, Canada

Tester F. Ashavaid, India

Chun Hang Au, Hong Kong, China

Rosa Ayala, Spain

Asfar Azmi, USA

Ulrike Bacher, Switzerland 
Aditya Bagrodia, USA

Nathanael G. Bailey, USA

Leonora Balaj, USA

Leomar Ballester, USA

Christian Barro, USA

Aaron Beasley, Australia

Gregory J. Berry, USA

Antonio Biondi, Italy

André Birgy, France

Gal Bitan, USA

Jorge C. G. Blanco, USA

Francisco Borrego, Spain

Veronika Borutinskaite, Lithuania

Constança F. P. C. Britto, Brazil

Diana I. Brixner, USA

Luca Campebel, France

F. David Carmona, Spain

Hiu Ting Chan, Japan

Aadel A. Chaudhuri, USA

Huan Chen, China

Yugo Chisaki, Japan

Rob Collin, the Netherlands

Curdin Conrad, Switzerland

Valeria Conti, Italy

Luis Antonio Corchete, Spain

Christopher L. Corless, USA

Lynsey Cree, New Zealand

Charbel Darido, Australia

Clara Dees, Germany

Klaus-Peter Dieckmann, Germany

Nicolas Dumaz, France

Eric Dumonteil, USA

Yogesh Dwivedi, USA

Frank Emmert-Streib, Finland

Dietmar Enko, Austria

Stepan M. Esagian, Greece

Henrik Falhammar, Sweden

Luo Fang, China

Harriet Feilotter, Canada

Renata Finelli, USA

María D. Flores-Chavez, Spain

Uta Flucke, the Netherlands

Lewis E. Fry, UK

Guoliang Fu, UK

Cristina B. García, Spain

Vassilis Georgoulias, Greece

Elena Gonzalez-Muñoz, Spain

Albert Grinshpun, Israel

Yueqing Gu, China

Allan J. Guimarães, Brazil

Peter Hagedorn, Denmark

Ferry Hagen, the Netherlands

Joelle A. Hallak, USA
Dora Hammerl, the Netherlands

Alan H. Handyside, UK

Alexandre Harlé, France

Steffen Heegaard, Denmark

Ellen Heitzer, Austria

Kevin A. Henry, Canada

Matthew I. Hiskens, Australia

Tham H. Hoang, USA

Marco H. Hofmann, Austria

Clive Hoggart, UK

Navin Horthongkham, Thailand

Shuiying $\mathrm{Hu}$, USA

Natalia Issaeva, USA

Moriya Iwaizumi, Japan

Iyare Izevbaye, Canada

Christopher M. Jackson, USA

Anders Jakobsen, Denmark

Edward J. Jarman, UK

Angie C. Jelin, USA

Carmen Jerónimo, Portugal

Zi-Bing Jin, China

Harm H. Kampinga, the Netherlands

Min-Jong Kang, USA

Antoine E. Karnoub, USA

Vera Kemp, the Netherlands

Nadine Kerr, USA

Mouhamad Khouja, Germany

Myungshin Kim, Republic of Korea

Deniz Kirac, Turkey

Susanne Klein-Scory, Germany

Susanne Kohl, Germany

Christos K. Kontos, Greece

Milena Kordalewska, USA

Sachin Kumar, India

Pappanaicken R. Kumaresan, USA

Elena Lastraioli, Italy

Nani M. Latar, Malaysia

Jen-Chieh Lee, Taiwan, Republic of China

Natasha B. Leighl, Canada

Chiuan Herng Leow, Malaysia

Jianguo Li, China

Massimo Libra, Italy

Juntang Lin, China

Nadine Lübke, Germany

Sonja Ludwig, USA

Ian M. MacDonald, Canada

Anil K. Madugundu, USA

Radhashree Maitra, USA

Karl-Johan Malmberg, Sweden

Michelangelo Mancuso, Italy

Teri Manolio, USA

Edoardo Marrani, Italy

Erin McClelland, USA 
Timothy J. Mead, USA

Estelle Menu, France

Steven W. Mes, the Netherlands

Sven Michel, Germany

Yuji Miura, Japan

Miriam Molina-Arcas, UK

Mark P. Molloy, Australia

Ana Morales, USA

Yoshitomo Morinaga, Japan

Jennifer J. D. Morrissette, USA

Florent Mouliere, the Netherlands

Cameron Mura, USA

Kei Murayama, Japan

Serge Muyldermans, Belgium

Shaik Mohammad Naushad, India

Jessica Nordlund, Sweden

Munier A. Nour, Canada

Raphael Nyaruaba, Kenya

Junna Oba, Japan

In-Jae Oh, Republic of Korea

Stacey S. O'Neill, USA

Nobuaki Ozawa, Japan

Nicolas Pallet, France

Christos Papaneophytou, Cyprus

Girolamo Pelaia, Italy

Charles Pilette, Belgium

Aurel Popa-Wagner, Australia

Constanza Potilinski, Argentina

Priscilla R. Prestes, Australia

Shengying Qin, China

Luis Quiñones, Chile

Damian T. Rieke, Germany

Jürgen Rödel, Germany

Paul Roepman, The Netherlands

Sabrina M. Ronen, USA

Cintia Roodveldt, Spain

Maria Rossing, Denmark

Alessandro Russo, Italy

Pierre Saintigny, France

Pierachille Santus, Italy

Vincent Sapin, France

Fernando Scaglia, USA

Jonathon Schmitz, USA

Andreas Schmutzler, Germany
Nicola Scichilone, Italy

Gianluca Serafini, Italy

Didik Setyo Heriyanto, Indonesia

Grégory Seumois, USA

Ankush Sharma, Norway

Julia C. Stingl, Germany

Hiromichi Suzuki, Japan

Silvia Tabano, Italy

Natalie Tan, Australia

Dai-Shi Tian, China

Vincent Timmerman, Belgium

Mario D. Toro, Poland

Federica Torricelli, Italy

Anubhav Tripathi, USA

Jan Trøst Jørgensen, Denmark

Şevin Turcan, Germany

Ilona Uzielene, Lithuania

Lia van der Hoek, The Netherlands

Léon C. van Kempen, The Netherlands

Karl Vandepoele, Belgium

Yiannis Vasilopoulos, Greece

Cecilia Vecoli, Italy

Tijl Vermassen, Belgium

Paola Viganò, Italy

Stefania Volpe, Italy

Roddy Walsh, the Netherlands

Chih-Yang Wang, Taiwan, Republic of China

Helen Wang, Sweden

Jinke Wang, China

Yuling Wang, Australia

Andrew J. Weickhardt, Australia

Eric Wickstrom, USA

Jae Kyung Won, Republic of Korea

Laura J. Wood, UK

Florian Wünnemann, Canada

Zhaolin Xu, Canada

Akira Yamada, Japan

Hidetaka Yamamoto, Japan

Chunzhang Yang, USA

Runkuan Yang, Norway

Yung-Li Yang, Taiwan, Republic of China

Zuocheng Yang, China

Weidong Yu, China

Rui Zhang, China

I am also very grateful to the members of the journal's Honorary Editorial Board, who have acted as peer reviewers and authors, and have provided guidance on journal content, policy and processes.

Springer Nature has continued to support the global response to COVID-19 by making all relevant content immediately and freely available. Indeed, Molecular Diagnosis \& Therapy has published a number of papers relevant to the COVID-19 pandemic that are free to access and can be found together as a journal collection (https://link.springer.com/journal/40291/ collections?filter=Open), including: 
- Analytical and Clinical Performance of Droplet Digital PCR in the Detection and Quantification of SARS-CoV-2. Kim, K.B., Choi, H., Lee, G.D. et al. Mol Diagn Ther 25, 617-628 (2021)

- A Prospective Evaluation of the Analytical Performance of GENECUBE ${ }^{\circledR}$ HQ SARS-CoV-2 and GENECUBE ${ }^{\circledR}$ FLU A/B. Kiyasu, Y., Akashi, Y., Sugiyama, A. et al. Mol Diagn Ther 25, 495-504 (2021).

- The Path Forward for COVID-19 Diagnostics. Usherwood, T., Zhang, L. \& Tripathi, A. Mol Diagn Ther 24, 637-639 (2020).

- Preliminary Analysis of B- and T-Cell Responses to SARS-CoV-2. Zhang, LX., Miao, SY., Qin, ZH. et al. Mol Diagn Ther 24, 601-609 (2020).

- Soluble Urokinase Plasminogen Activator Receptor: A Biomarker for Predicting Complications and Critical Care Admission of COVID-19 Patients. Chalkias, A., Mouzarou, A., Samara, E. et al. Mol Diagn Ther 24, 517-521 (2020).

- The Potential Role of Smartphone-Based Microfluidic Systems for Rapid Detection of COVID-19 Using Saliva Specimen. Farshidfar, N., Hamedani, S. Mol Diagn Ther 24, 371-373 (2020).

In terms of other important company initiatives, the Springer Nature 'Sustainable Development Goals (SDG) Programme' is aiming to connect researchers who are tackling the world's toughest challenges with practitioners in policy and business, while the 'Women in Science' initiative aims to help empower more women scientists to achieve scientific excellence. Finally, I am delighted to inform you that Springer Nature is now carbon neutral for its direct operations (offices, fleet and flights) and earlier this year signed The Climate Pledge, making a commitment to be Net Zero carbon by 2040.

Returning to Molecular Diagnosis \& Therapy, the editorial program for 2022 is well under way, and I am looking forward to bringing you many high-quality and authoritative articles over the coming year.

I thank you for your continued support.

With best wishes,

Alison Fitches 